\title{
Trace Anomaly and Backreaction of the Dynamical Casimir Effect
}

\author{
M.R. Setare * \\ Physics Dept. Inst. for Studies in Theo. Physics and Mathematics(IPM) \\ P. O. Box 19395-5531, Tehran, IRAN \\ and \\ Department of Science, Physics group, Kordestan University, Sanandeg, Iran \\ and \\ Department of Physics, Sharif University of Technology, Tehran, Iran
}

October 25, 2018

\begin{abstract}
The Casimir energy for massless scalar field which satisfies priodic boundary conditions in two-dimensional domain wall background is calculated by making use of general properties of renormalized stress-tensor. The line element of domain wall is time dependent, the trace anomaly which is the nonvanishing $T_{\mu}^{\mu}$ for a conformally invariant field after renormalization, represent the back reaction of the dynamical Casimir effect.
\end{abstract}

\footnotetext{
*E-mail: rezakord@yahoo.com
} 


\section{Introduction}

In the semiclassical approximation theory of quantum gravity we are involved with calculation the expectation value of energy momentum tensor in special vacuum, [1]. However the usual expression for the stress tensor includes singular products of the field operators for stress tensor.Renormalization theory of the stress tensor claims to solve this problem, but it must be mentioned that the usual scheme of renormalization includes complexity and somewhat ambiguity. For instance, there is no conceptual support for a local measure of energy momentum of some given state without any reference to any global structure. We know in this frame energy is source of gravity and we are not allowed to subtract any unwanted part of energy even though it is infinite. So to consider the back reaction effect of the quantum field on the gravitational field, we must find a more elaborate renormalization scheme in which the dynamics of gravitational field is a vital component. In original Casimir effect discovered in 1948 by H.B.G Casimir [2] we are concerned about force and energy, but we are not usually interested in dynamics of the gravitational field. Even in many cases in curved boundary problems, energy is not our main concern. Because of unphysical nature of boundary condition the energy diverges approaching to curved boundary [3]. The Casimir effect can be viewed as a polarization of vacuum by boundary conditions and external fields, such as gravitational field. In the present paper we are going to consider a simple example in which these two types of sources for vacuum polarization are present.There is several methods for calculating Casimir energy. For instance, we can mention mode summation, Green's function method [4], heat kernel method [5, 6]along with appropriate regularization schemes such as point separation [7],[8] dimensional regularization [9], zeta function regularization [10, 11, 12]. But it must be remarked that practically all of the methods are successful only for boundary conditions with high symmetry in flat space time. In fact we don't have any general procedure for renormalizing stress tensor in gravitational background with arbitrary boundary ( to see general new methods to compute renormalized one-loop quantum energies and energy densities Ref $[13,14])$.

In the static situation, the disturbance of the quantum state induces vacuum energy and stress, but no particles are created. The creation of particles from the vacuum takes place due to the interaction with dynamical external constraints. For example the motion of a single reflecting boundary (mirror) can create particles [1], the creation of particles by time-dependent external gravitational field is another example of dynamical external constraints. In two-dimensional space-time and for conformally invariant fields the problem with dynamical boundaries can be mapped to the corresponding static problem and hence allows a complete study (see Refs. [1, 15] and references therein).

It has been shown $[16,17]$ that particle creation by black hole in four dimension is as a consequence of the Casimir effect for spherical shell. It has been shown that the only existence of the horizon and of the barrier in the effective potential is sufficient to compel the black hole to emit black-body radiation with temperature that exactly coincides with the standard result for Hawking radiation. In [17], the results for the accelerated-mirror have been used to prove above statement.

In this paper the Casimir stress tensor for scalar field which satisfies periodic boundary conditions in two dimensional analog of domain wall space time, is calculated. In this case we do not need the boundaries. For the purpose of describing the Casimir effect in the one-dimensional cavity by the moving mirrors, one can consider a massless field 
in the one-dimensional finite space with two boundaries. The motion of the boundaries generally mixes the energy levels of the system. However, when the motion of the size of cavity is adiabatic, there are no transitions among the energy levels [18]. For this purpose we replace the spatial size of cavity with $S^{1}$ space, therefore the scalar field required to satisfy the periodic boundary condition. The two dimensional domain wall space time which we have considered is the Robertson-Walker type metric, we can regard the size of $S^{1}$ as the scale factor of the metric. The Casimir stress tensor is obtained by imposing only general requirements which is discussed in section 2 .We show direct relation between trace anomaly and Casimir effect,although we have been aware of role of anomalous trace in gravitational background such as Hawking effect [19]. Knowing of Casimir energy in flat space and trace anomaly help us to calculate renormalized stress tensor. To see similar calculation in background of static domain wall and two-dimensional Schwarzschild black hole, refer to [20, 21], see also [22]for Casimir effect in 2D stringy black hole backgrounds.

\section{General properties of stress tensor}

In semiclassical framework for yielding a sensible theory of back reaction Wald [23] has developed an axiomatic approach. There one tries to obtain an expression for the renormalized $T_{\mu \nu}$ from the properties (axioms) which it must fulfill. The axioms for the renormalized energy momentum tensor are as follow:

1-For off-diagonal elements standard results should be obtained.

2-In Minkowski space time standard results should be obtained.

3 -Expectation values of energy momentum are conserved.

4-Causality holds .

5-Energy momentum tensor contains no local curvature tensor depending on derivatives of the metric higher than second order.

Two prescriptions that satisfy the first four axioms can differ at most by a conserved local curvature term. Wald, [24], showed any prescription for renormalized $T_{\mu \nu}$ which is consistent with axioms 1-4 must yield the given trace up to the addition of the trace of conserved local curvature .It must be noted (that trace anomalies in stress-tensor,that is, the nonvanishing $T_{\mu}^{\mu}$ for a conformally invariant field after renormalization) are originated from some quantum behavior [25]. In two dimensional space time one can show that a trace-free stress tensor can not be consistent with conservation and causality if particle creation occurs. A trace-free ,conserved stress tensor in two dimensions must always remain zero if it is initially zero. One can show that the "Davies-Fulling-Unruh" [26] formula for the stress tensor of scalar field which yield an anomalous trace,$T_{\mu}^{\mu}=\frac{R}{24 \pi}$, is the unique one which is consistent with the above axioms. In four dimensions, just as in two dimensions, a trace-free stress tensor which agrees with the formal expression for the matrix elements between orthogonal states can not be compatible with both conservation laws and causality . It must be noted that, as showed Wald[24], with Hadamard regularization in massless case axiom(5) can not be satisfied unless we introduce a new fundamental length scale for nature. Regarding all these axioms,thus, we are able to get an unambiguous prescription for calculation of stress tensor. 


\section{Vacuum expectation values of stress tensor}

Vilenkin [27] and Isper and Sikvie [28] have solved Einstein's equations in the presence of a planar domain wall by approximating the stress energy of the wall as that of an infinitely thin plane with positive energy density and negative, homogeneouse, and isotropic pressure in the plane of the wall. The stress- energy tensor is taken to be

$$
T_{\mu}^{\nu}=\sigma \delta(z) \operatorname{diag}(1,-1,-1,0),
$$

where $\sigma$ is the mass per unite area of the wall and the $z$ axis is perpendicular to the wall. In suitable coordinates, the metric takes the form

$$
d s^{2}=e^{-k|z|}\left[-d t^{2}+d z^{2}+e^{k t}\left(d x^{2}+d y^{2}\right)\right] .
$$

The geometry of hypersurfaces $z=$ const, is that of $(2+1)$-dimensional de Sitter space. The $(t, z)$ part of metric describes a $(1+1)$-dimensional Rindler space.

Now, just for the sake of simplicity, we consider two dimensions in which

$$
d s^{2}=-d t^{2}+e^{k t} d x^{2},
$$

where we define the above domain wall- type metric on the space-time $R \times S^{1}$, and $0 \leq x \leq l$, where a dimensional constant $l$ is the standard space size and $e^{\frac{k t}{2}}$ is the scale factor. We can rewrite the metric into the conformal flat form by general coordinate transformation

$$
d s^{2}=-d t^{2}+e^{k t} d x^{2}=-c(\eta)\left(d \eta^{2}-d x^{2}\right),
$$

where we have introduced a new coordinate $\eta$ such that

$$
d \eta=e^{-\frac{k t}{2}} d t, \quad c(\eta)=e^{k t} .
$$

From now on, our main goal is to determine a general form of conserved energy-momentum tensor, regarding trace anomaly for the metric Eq.(3). Once we consider a massless scalar filed in the space $S^{1}$, the scalar filed is required to satisfy the periodic boundary condition. When we quantize the scalar filed, the conformal anomaly appears in general. The quantum effects leads to the motion of scale factor [29], in another word the motion of the scale factor is the back reaction of the Casimir effects.

For the non -zero Christoffel symbols of the metric Eq.(4)we have ;

$$
\Gamma_{x x}^{\eta}=\Gamma_{x \eta}^{x}=\Gamma_{\eta \eta}^{x}=\Gamma_{\eta \eta}^{\eta}=\frac{\dot{c}}{2 c}
$$

Then the conservation equation takes the following form

$$
\begin{aligned}
& \partial_{\eta} T_{x}^{\eta}+\Gamma_{x \eta}^{x} T_{x}^{\eta}-\Gamma_{x x}^{\eta} T_{\eta}^{x}=0 \\
& \partial_{\eta} T_{\eta}^{\eta}+\Gamma_{x \eta}^{x} T_{\eta}^{\eta}-\Gamma_{x \eta}^{x} T_{x}^{x}=0
\end{aligned}
$$

in which,

$$
T_{\eta}^{x}=-T_{x}^{\eta} \quad T_{x}^{x}=T_{\beta}^{\beta}-T_{\eta}^{\eta}
$$

and $T_{\beta}^{\beta}$ is anomalous trace in two dimension.Using the Eqs. $(6-8)$ it could be shown that

$$
\frac{d\left(T_{x}^{\eta} c(\eta)\right)}{d \eta}=0
$$


and

$$
\frac{d\left(T_{\eta}^{\eta} c(\eta)\right)}{d \eta}=\frac{\dot{c}(\eta)}{2} T_{\beta}^{\beta}
$$

Then Eq.(10) leads to:

$$
T_{x}^{\eta}=\alpha^{\prime} c^{-1}(\eta)
$$

where $\alpha^{\prime}$ is the constant of integration. The solution of Eq.(11) might be written in the following form

$$
T_{\eta}^{\eta}(\eta)=(H(\eta)+\zeta) c^{-1}(\eta)
$$

where

$$
H(\eta)=1 / 2 \int_{\eta_{0}}^{\eta} T_{\beta}^{\beta} \dot{c}\left(\eta^{\prime}\right) d \eta^{\prime}
$$

and anomalous trace is given by [1]

$$
T_{\beta}^{\beta}=\frac{R}{24 \pi}=\frac{k^{2}}{48 \pi} .
$$

When we chose $\eta_{0}=0$, the function $H(\eta)$ is given by

$$
H(\eta)=\frac{k^{2}}{96 \pi}(c(\eta)-1)
$$

Using the Eqs. (9), (12)and (13) it can be shown that energy momentum tensor takes the following form in $(\eta, x)$ coordinates. So we have most general form of stress tensor field in our interesting background.

$$
T_{\nu}^{\mu}(\eta)=\left(\begin{array}{cc}
H(\eta) c^{-1}(\eta) & 0 \\
0 & T_{\beta}^{\beta}-c^{-1}(\eta) H(\eta)
\end{array}\right)+c^{-1}\left(\begin{array}{cc}
\zeta & \alpha^{\prime} \\
-\alpha^{\prime} & -\zeta
\end{array}\right)
$$

Now we are going to obtain two constants $\alpha^{\prime}$ and $\zeta$ by imposing the second axiom of renormalization scheme. So when we put $k=0$, we reach the special case of flat spacetime. The type of boundary condition which we choose is periodic boundary condition $\phi(x, t)=\phi(x+2 n \pi, t)$. This is easiest generalization of Minkowski space quantum field theory to non-trivial topological structures in a locally flat space-time. This space-time is $R^{1} \times S^{1}$, which has two-dimensional Minkowski space line element, but the spatial point $x$ and $x+2 \pi a$ are identified, where $a$ is the radius of circle $S^{1}$. The cartesian components of the vacuum expectation values of the stress-tensor are as following [1]

$$
\begin{gathered}
\rho=<0\left|T_{\eta \eta}\right| 0>=\frac{-1}{24 \pi a^{2}}, \\
<0\left|T_{\eta x}\right| 0>=0, \\
P=<0\left|T_{x x}\right| 0>=\frac{-1}{24 \pi a^{2}} .
\end{gathered}
$$

Comparing Eqs.(18-20) with Eq. (17) we obtain

$$
\zeta=\frac{-1}{24 \pi a^{2}}, \quad \alpha^{\prime}=0
$$

Thus we have obtained the energy momentum tensor as direct sum of two terms; first term which present the vacuum polarization in gravitational background in the absence of 
boundaries, and second term which come from periodic boundary condition or in another word due to the nontrivial topology of space-time. (See the Ref.[20, 21, 22] for similar calculation in another $(1+1)$ dimensional gravitational background).

$$
<T_{\nu}^{\mu}>=<T_{\nu}^{(g) \mu}>+<T_{\nu}^{(t) \mu}>
$$

where $\left\langle T_{\nu}^{(g) \mu}>\right.$ and $\left\langle T_{\nu}^{(t) \mu}>\right.$ stand for gravitational and topological parts respectively. It should be noted that the trace anomaly has a contribution just in the first term < $T_{\nu}^{(g) \mu}>$, which comes from the background effect not the topological one. However, it has a contribution in the total Casimir energy-momentum tensor.

The gravitational part pressure are given by

$$
P^{(g)}=-<T_{1}^{(g) 1}>=\frac{-k^{2}}{96 \pi}\left(1+e^{-k t}\right),
$$

as is clear this pressure come from trace anomaly, represent the back-reactional force of the dynamical Casimir effect. The vacuum topological part pressure are as following

$$
P^{(t)}=-<T_{1}^{(t) 1}>=\frac{-1}{24 \pi a^{2}} e^{-k t} .
$$

Therefore the total pressure naturally contains both the ordinary Casimir energy term and the back-reaction term of the dynamical Casimir effect.

\section{Conclusion}

We have found the renormalized energy-momentum tensor for massless scalar field on background of $1+1$ dimensional domain wall with periodic conditions, by making use of general properties of stress tensor only. An essential point of our approach is replacing the mirror separation into the size of space $S^{1}$ in the adiabatic approximation. The motion of the cavity size is described by varying the radius of $S^{1}$ in time. That is, the mirror separation is described by the scale factor of Robertson-Walker type metric. The time evolution of the scale factor can be regarded as the space-time $R \times S^{1}$ with gravity. The trace anomaly is especially important in the special case that the background spacetime is conformally flat. If the quantum field is also conformally invariant, then we have a conformally trivial situation. In this case, it turns that anomalous trace determines the entire stress-tensor once out the quantum state has been specified $[1,30]$. We propose that if we know the stress tensor for a given boundary in Minkowski space-time, the Casimir effect in gravitational background can be calculated. We have found direct relation between trace anomaly and total Casimir energy. The conformal anomaly term represent the back reaction of the dynamical Casimir effect. From the resultant energy-momentum tensor, we have obtained the dynamical vacuum pressure. The pressure (dynamical Casimir force) includes the back-reactional force of the dynamical Casimir effect. The dynamical Casimir force was confirmed to be attractive and always stronger than the topological Casimir force.

\section{References}

[1] N. D. Birrell and P. C. W. Davies "Quantum fields in curved space". (Chambridge University press,1982). 
[2] H. B. G. Casimir, proc. kon. ned. akad. wetensch 51,793(1948).

[3] D. Deutsch and P. Candelas. Phys. Rev. D20, 3063(1979).

[4] G. Plunien, B. Muller, W. Greiner, Phys. Rep. 134, 87(1986).

[5] Klaus. Kirsten, SPECTRAL FUNCTIONS IN MATHEMATICS AND PHYSICS, (Leipzig, Max Planck Inst.). 2001. 382pp.

[6] K. Bormann and Antonsen "The Casimir effect of curved spacetime", hepth/9608142.

[7] S. M. Christensen, Phys. Rev. D14, 2490(1976); 17, 946,(1978).

[8] S. L. Adler, J. Lieberman and Y. J. Ng, Ann. Phy. (N.Y) 106, 279(1977).

[9] S. Deser, M. J. Duff and C. J. Isham, Nucl. Phys B11, 45 (1976),see also D. M. capper and M. J. Duff, Nuovo Cimento 23A, 173(1974); Phys. Lett.53A, 361(1975).

[10] S. W. Hawking, Commun. Math. Phys. 55, 133(1977).

[11] E. Elizalde, S. D. Odintsov, A. Romeo, A. A. Bytsenko and S. Zerbini, zeta regularization techniques with applications(World Scientific, Singapore, 1994).

[12] E. Elizalde, Ten physical applications of spectral zeta functions, lecture notes in physics, (Springer-Verlage, Berlin, 1995).

[13] N. Graham, R. L. Jaffe, V. Khemani, M. Quandt, M. Scandurra, H. Weigel, Nucl.Phys.B645, 49, (2002).

[14] N. Graham, R. L. Jaffe, V. Khemani, M. Quandt, M. Scandurra, H. Weigel, hepth/0207205.

[15] K. A. Milton, The Casimir Effect: Physical Manifestations Of Zero-Point Energy, World Scientific (2001).

[16] R. M. Nugayev, V. I. Bashkov, Phys. Lett. 69A, 385(1979)

[17] R. M. Nugayev, Phys. Lett. 91A, 216(1982).

[18] E. Sassaroli, Y. N. Srivastava, and A. Widom, Phys. Rev. A50, 1027 (1994).

[19] S. M. Christensen and S. A. Fulling, Phys. Rev. D15, 2088(1977).

[20] M. R. Setare and A. H. Rezaeian. Mod. Phys. Lett. A15, 2159(2000).

[21] M. R. Setare. Class.Quant.Grav. 18, 2097 (2001).

[22] T. Christodoulakis, G.A. Diamandis, B.C. Georgalas, E.C. Vagenas, Phys.Rev. D64, 124022, (2001).

[23] R. M. Wald, Commun. Math. Phys. 54, 1(1977).

[24] R. M. Wald, Phys. Rev. D17, 1477,(1978). 
[25] S. Coleman and R. Jackiw, Ann. Phys. (NY)67, 552(1971).

[26] D. C. W. Davies, S. A. Fulling and W. G.Unruh, Phys. Rev D13, 2720 (1976). and also see A. Z. capri, M. Kobayashi and D. J. Lamb Class. Quant. Grav. 13, 179(1996).

[27] A. Vilenkin, Phys. Lett. 133B, 177(1983).

[28] J. Ipser and P. Sikivie, Phys. Rev. D30, 712 (1984).

[29] Y. Nagatani and Kei Shigetomi. Phys.Rev.A62 (2000) 022117.

[30] L. S. Brown and J. P. Cassidy. Phys. Rev. D16, 1712 (1977). 